\title{
Effects of Ply Stacking Sequence in 3D Fiber Reinforced Foam Core Sandwich Structures with Defects
}

\author{
Zachary T. Kier ${ }^{*}$ and Anthony M. Waas ${ }^{*}$ \\ Composite Structures Laboratory, Department of Aerospace Engineering \\ University of Michigan, Ann Arbor, MI. \\ and \\ Chad Foerster ${ }^{*}$ Jacob I. Rome $e^{\S}$, and Vinay K. Goyal ${ }^{* *}$ \\ The Aerospace Corporation, El Segundo, CA 90245
}

\begin{abstract}
Foam core sandwich composites are widely used in primary structural components of launch vehicles and spacecraft. These structures exhibit complex failure modes and are highly sensitive to butt-joints, core mismatches, impact damage, voids, and facesheet delaminations. 3D Fiber Reinforced Foam Cores (3DFRFC) represent a new class of core material designed to replace standard foam core in future aerospace structures. An analysis program is being developed to investigate the effect of ply stacking sequence on debond buckling in 3DFRFC structures with defects. The detailed microstructure is modeled using the embedded element method to investigate the local interaction between the facesheet and the core. Linear and nonlinear buckling analysis was used to understand the sensitivity of the predicted buckling load to the ply stacking sequence. Preliminary results show that the effect of ply stacking sequence on the buckling load is consistent across variations to the core types, modeling methods, and defect sizes investigated.
\end{abstract}

\section{Introduction}

Sandwich composites offer key advantages in automotive and aerospace applications including reduction in weight over metals currently used. A current engineering challenge of utilizing sandwich composite structures is quantifying their ability to tolerate damage, particularly in launch vehicles and spacecraft, where mission assurance is critical to mitigating cost from loss or failure, technological set-backs, and potential risk to human life. The strength of sandwich composites can be reduced through many mechanisms, including debonds between a facesheet and the core. Debonds of sufficient sizes, which are typically introduced during manufacturing of foam core composite sandwich structures, could become critical and lead to

\footnotetext{
*NASA Space Technology Research Fellow, Department of Aerospace Engineering, 1320 Beal Avenue, Ann Arbor, MI 48109, AIAA Student Member, ztkier@umich.edu.

${ }^{\dagger}$ Felix Pawlowski Collegiate Professor of Aerospace Engineering, Department of Aerospace Engineering, AIAA Fellow.

${ }^{*}$ Member of Technical Staff, Structures Department, AIAA Member.

${ }^{\S}$ Senior Engineering Specialist, Structures Department, AIAA Member.

${ }^{* *}$ Engineering Specialist, Structures Department, AIAA Member.
}

American Institute of Aeronautics and Astronautics 
catastrophic failure. These debonds can result from impact damage, embedded foreign objects, use of poor bonding agents, or surface preparation issues.

Recently, new core materials have been developed that have the potential to affect the damage tolerance of sandwich composites. One class of core material being considered may alter their damage tolerance through the use of three-dimensional, truss-like networks of reinforcing fibers inside a lightweight foam core. This overall class of 3D Fiber Reinforced Foam Core (3DFRFC) is defined to be quite broad and encompasses a substantial design envelope of core material with freedom for the tailorability of the effective core properties. The design space of 3DFRFCs can be explored through the selection of the base foam (material, density, thickness), the geometry of the truss structure, the type of reinforcing fibers used (carbon, glass, Kevlar ${ }^{\circledR}$, Spectra ${ }^{\circledR}$, etc.), and the method of manufacture (resin infusion, prepreg fiber insertion). Examples of this emerging class of core materials include NidaFusion ${ }^{1}$, TYCOR ${ }^{\circledR}{ }^{2}$, and K-Cor ${ }^{3}$, Figure 1 .

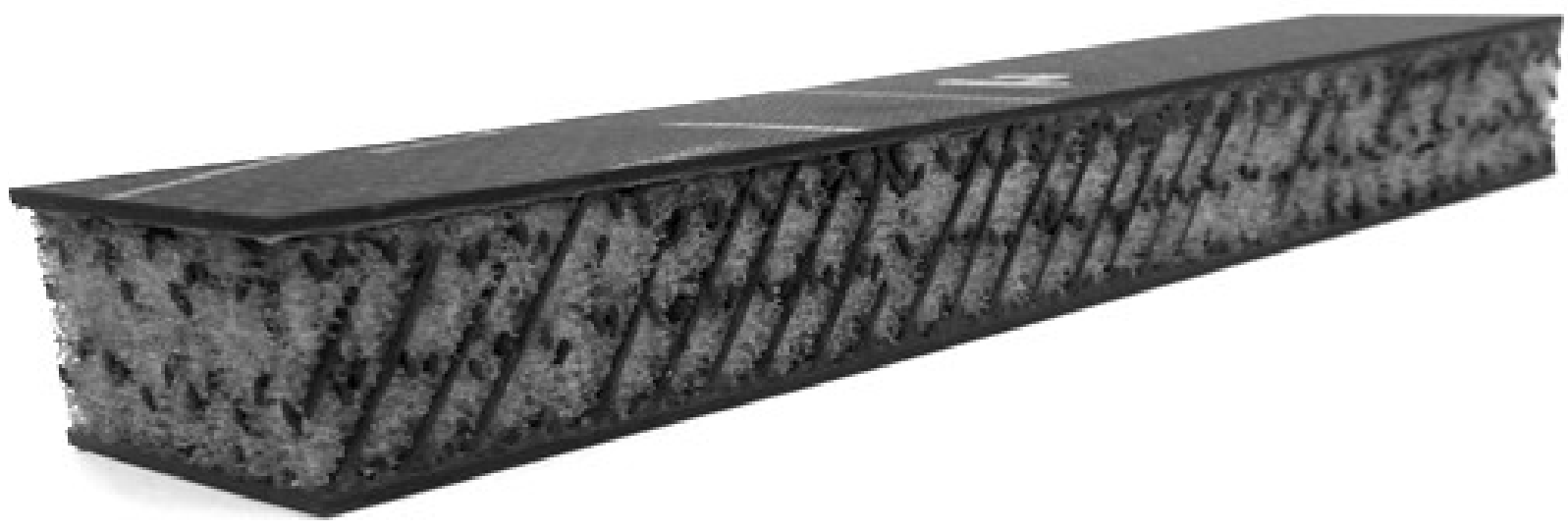

Figure 1. Sandwich composite made with a 3D fiber reinforced foam core. (Image not to scale.)

The truss structure of the 3D fiber network provides added paths for load transfer and acts to impede crack propagation within the foam core. The stiffness and strength of these threedimensionally reinforced sandwich composites become exceedingly difficult to predict as a result of the added load paths. In addition, the relatively coarse architecture of 3DFRFCs can lead to local variations due to the interactions of the facesheet and the reinforcements within the core.

Previous studies have highlighted the role of localized facesheet buckling in the failure of reinforced and unreinforced foam core sandwich structures with facesheet-to-core debonds subjected to edgewise compression (EWC) loading ${ }^{4,5}$. These studies demonstrated that for larger defects the specimen failure was driven by the local instability of the facesheet in the unbonded region. While the extent of the failure differed between the reinforced and unreinforced foam core samples, Figure 2, the initiating mode in both cases was localized buckling of the facesheet. While these studies looked at the global failure of the samples, no investigation was conducted to examine the sensitivity of this failure mode to the ply stacking sequence of the facesheets or how the local interaction of the reinforcement with the facesheet affects the onset of the local buckling. 


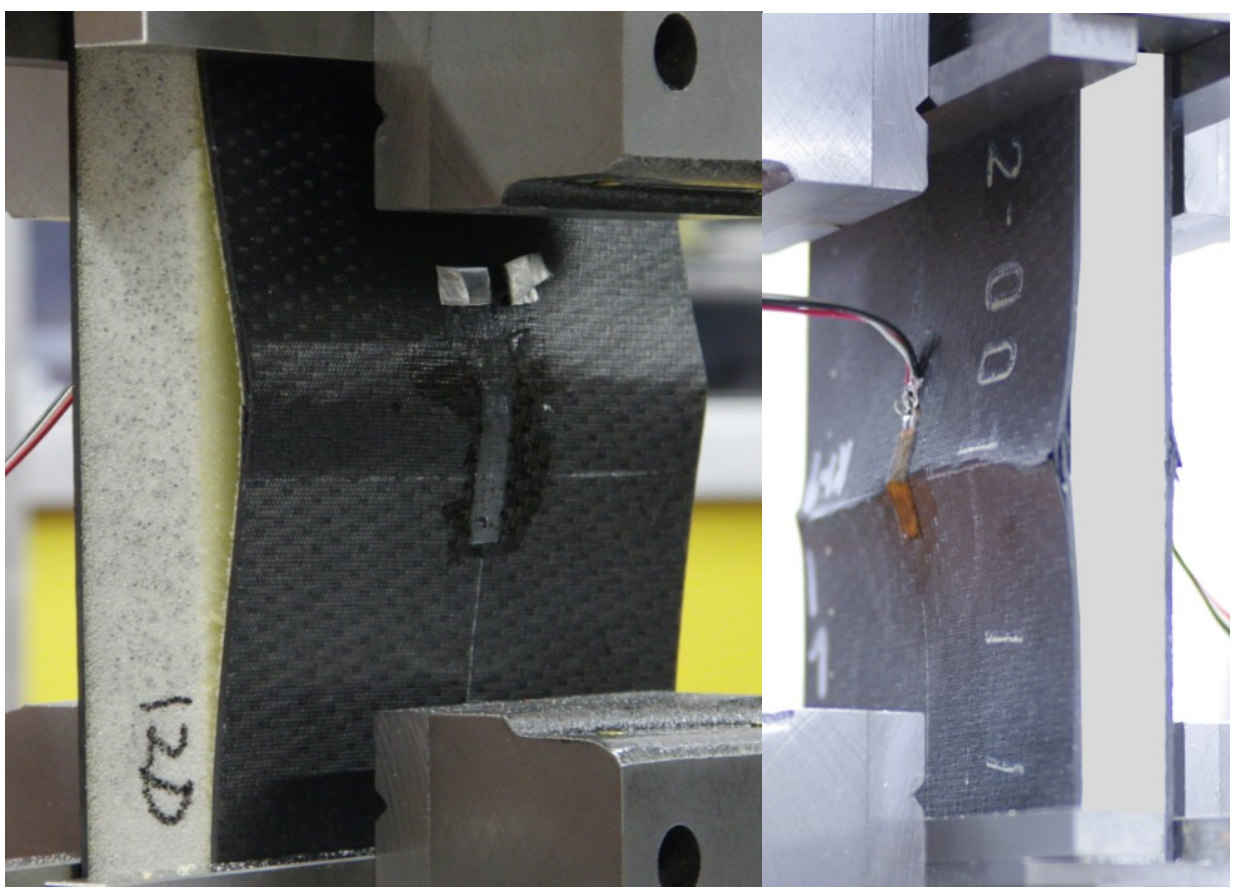

Figure 2. Failed EWC samples with unreinforced foam (left) and reinforced foam (right). (Details of reinforcement geometry removed. Images used with the permission of The Aerospace Corporation.)

This research aims to investigate the effect of the local interactions and ply stacking sequence on facesheet stability in reinforced and unreinforced foam core composite sandwich structures with facesheet-to-core debonds. This includes the implementation of a detailed microstructure model to better understand the interaction and behavior of the 3DFRFC material with changes in ply stacking sequence. Due to the complexity of the 3DFRFC, this investigation is focused on analysis of test specimen geometry. Analytical results that highlight unique behavior will subsequently be validated with follow-on experiments.

\section{Modeling 3DFRFC Microstructure and Edgewise Compression Samples}

Modeling efforts have been focused on developing tools to allow for the detailed modeling of the microstructure of the 3DFRFC. Using the commercial code Abaqus, this effort has resulted in a parametric Abaqus script that automates the creation of the microstructure geometry for the reinforcing fibers based on the geometric parameters of the microstructure and the size of the specimen to be modeled, Figure 3 and Figure 4. Note the reinforcing geometry is modeled using beams; however, some images show the fibers with rendered thickness for clarity. This script incorporates details of the bonding ends of the reinforcement into the geometry generated, shown in the inset in Figure 4. The geometry generated by this script was utilized to develop the embedded element models of the edgewise compression sample configurations. 


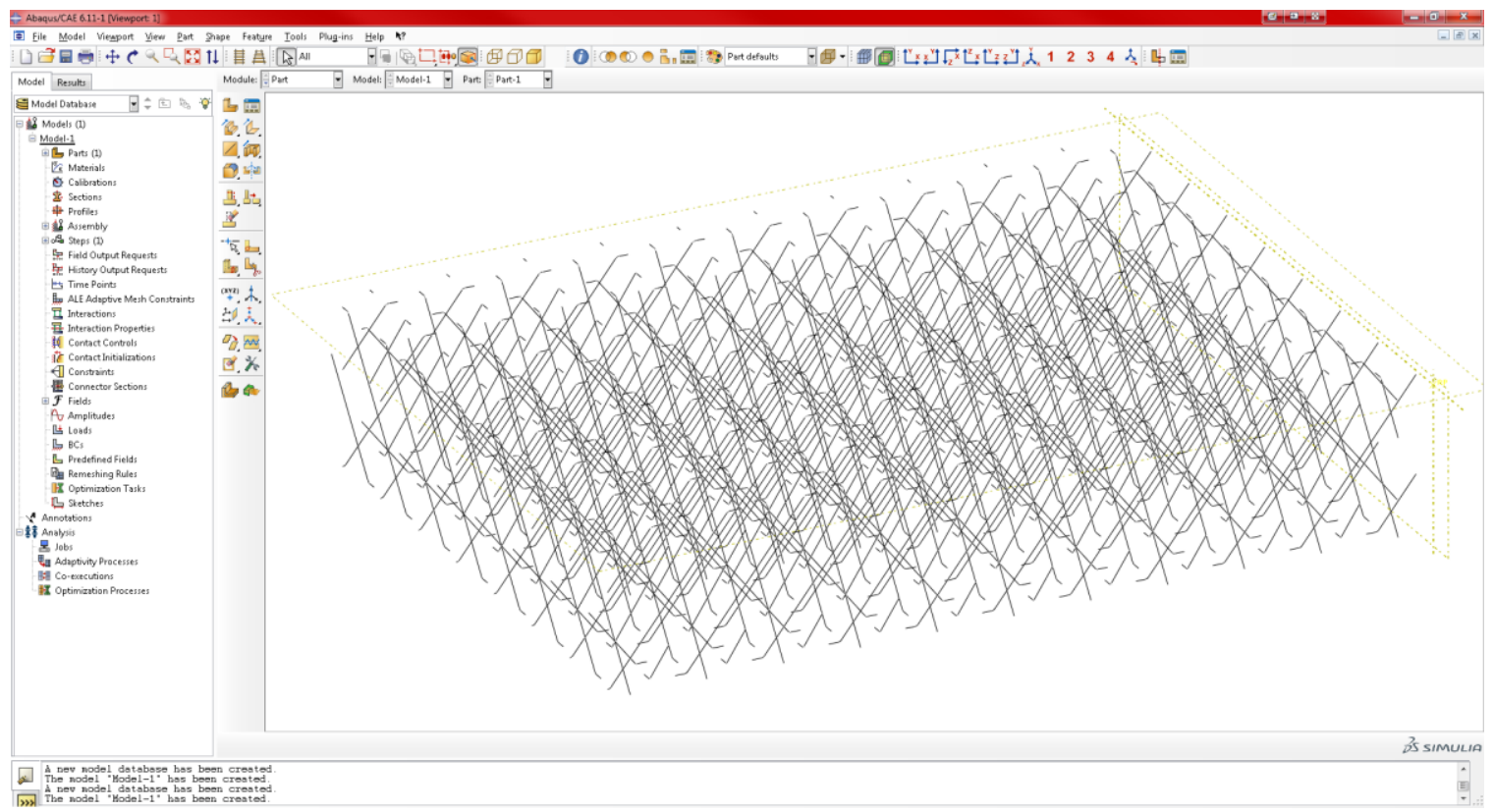

Figure 3. A screen capture of geometry output by parametric Abaqus script for a $7 \times 10$ unit cell model with $38^{\circ}$ reinforcement.

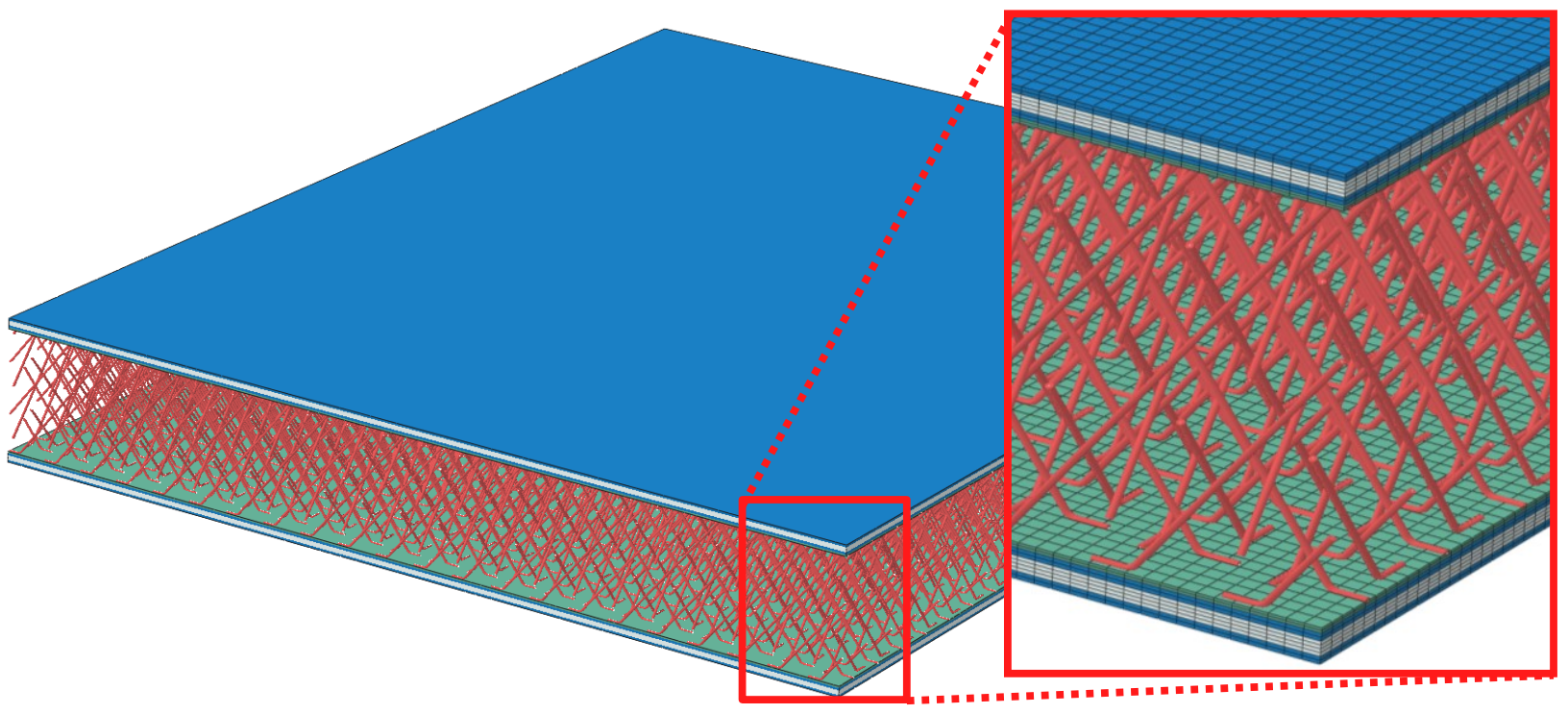

Figure 4. 27 x 22 unit cell model of a 3DFRFC sandwich composite with foam removed. (Image not to scale.)

The material system chosen for this investigation is an 8-harness satin weave IM7/8552 carbon/epoxy prepreg for the facesheets and a 0.75 inch thick $12 \mathrm{lb} / \mathrm{ft}^{3} 3 \mathrm{DFRFC}$ for the core. A 0.75 inch thick $12 \mathrm{lb} / \mathrm{ft}^{3}$ unreinforced foam core is also modeled for comparison purposes. AF 191 film adhesive is used to bond the facesheets to the core. All models contain 4 ply facesheets with permutations on the baseline ply stacking sequence $\left[0^{\circ} / \pm 45^{\circ} / \pm 45^{\circ} / 0^{\circ}\right]$. All models will contain two $\left[0^{\circ} / 90^{\circ}\right]$ plies and two $\left[ \pm 45^{\circ}\right]$ plies; however, the order of the plies will be varied. The upper and lower facesheets will remain symmetric about the sandwich core to keep the global sandwich balanced. 
The evaluation of the EWC configurations was performed with a finite element analysis using Abaqus. The facesheet plies and adhesive layers were individually modeled with brick elements. The facesheet and unreinforced foam core properties were measured through testing at The Aerospace Corporation ${ }^{6}$, while the mechanical properties of the film adhesive were obtained from vendor data ${ }^{7}$. The 3 DFRFC is modeled utilizing two different methods: homogenized orthotropic and embedded element. The homogenized orthotropic model takes advantage of recently developed micromechanics models ${ }^{8}$ for metallic and pin reinforced foams to derive the homogenized orthotropic elastic properties for the 3DFRFC based on its specific microstructure and material composition. The embedded element method utilizes the aforementioned Abaqus script to model the discrete reinforcing pins as beams within the solid foam mesh. The embedded model allows for investigation of the local microstructure interaction that is not captured by the homogenized method and will provide additional information for future failure analyses.
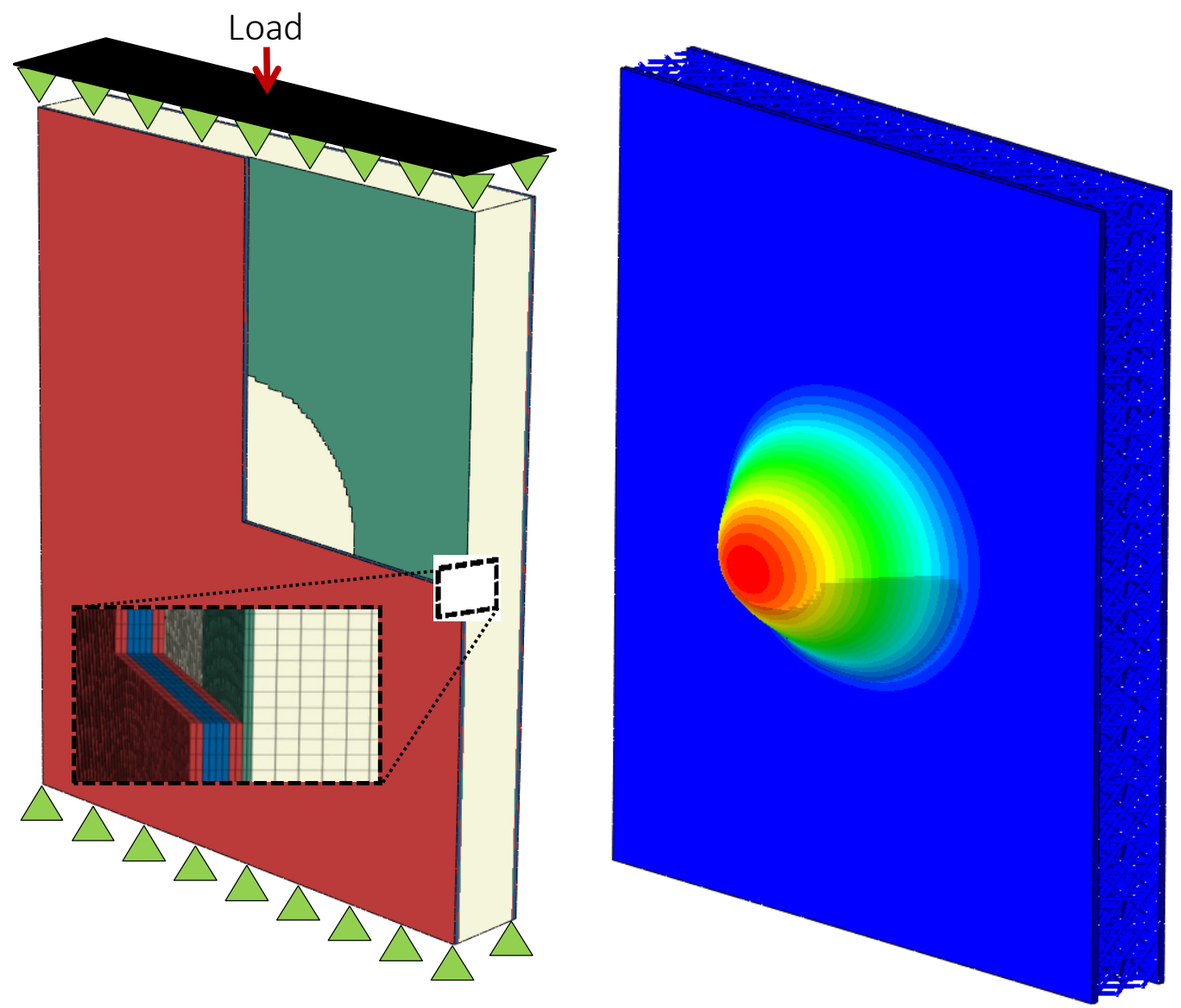

Figure 5. Model configuration shown with portion of facesheet removed, left, and representative buckling mode shape shown with foam removed, right.

The same structured base mesh was utilized for all of the cases and was sufficiently refined to provide a converged solution and adequate detail for the embedded element model resulting in a base mesh of approximately 5 million degrees-of-freedom. The model used contains approximately 600 representative unit cells (RUCs). It is important to note that RUC in this analysis is defined strictly from the minimum geometric unit needed to capture the repeating structure of the 3DFRFC. A total of 6 permutations of the baseline facesheet ply stacking 
sequence are possible and were investigated: $\left[0^{\circ} / \pm 45^{\circ} / \pm 45^{\circ} / 0^{\circ}\right]$ (Baseline), $\left[ \pm 45^{\circ} / 0^{\circ} / 0^{\circ} / \pm 45^{\circ}\right]$, $\left[0^{\circ} / 0^{\circ} \pm 45^{\circ} / \pm 45^{\circ}\right],\left[ \pm 45^{\circ} / \pm 45^{\circ} / 0^{\circ} / 0^{\circ}\right],\left[0^{\circ} / \pm 45^{\circ} / 0^{\circ} / \pm 45^{\circ}\right]$, and $\left[ \pm 45^{\circ} / 0^{\circ} / \pm 45^{\circ} / 0^{\circ}\right]$. For all cases the facesheet-to-core debond was created by removing a circular region of the adhesive layer, Figure 5. Three different circular defect sizes were investigated for each of the three core models and six ply stacking sequences resulting in a total of 54 models. The three defect diameters(d) were sized relative to the specimen width(w): $d=1 / 4 \mathrm{w}, \mathrm{d}=3 / 8 \mathrm{w}$, and $\mathrm{d}=1 / 2 \mathrm{w}$. Linear buckling analysis showed that all models exhibited localized faceheet buckling as the primary mode, similar to the one shown in Figure 5. The mode corresponding to the smallest linear buckling load had a positive out-of-plane displacement amplitude, thus precluding the possibility of contact buckling (that may occur for higher modes or differing boundary conditions). Issues related to contact buckling of debonds are addressed in Comiez et al $^{9}$.

\section{Effects of Ply Stacking on Predicted Linear Buckling Load}

Linear buckling analysis was conducted on the unreinforced foam core model first to provide a foundation for evaluating the behavior of the 3DFRFC models. All of the buckling loads are normalized by the linear buckling load for the unreinforced foam core sandwich with baseline ply stacking $\left[0^{\circ} / \pm 45^{\circ} / \pm 45^{\circ} / 0^{\circ}\right]$ and $\mathrm{d}=1 / 2 \mathrm{w}$ in order to allow for direct comparison of the various models. The results for the unreinforced foam core are given in Figure 6 . These results highlight the influence of the ply stacking sequence on the predicted buckling load. While the buckling loads increase as the defect diameter is decreased, the relative effect of changing the ply stacking sequence is consistent for all sizes. A nearly identical trend was observed for the two 3DFRFC models, Figure 7 and Figure 8. Both the homogenized and embedded 3DFRFC models predict an increase in buckling load as compared to the unreinforced foam core models. The embedded model exhibits a lower value compared to the homogenized model likely due to its ability to account for the interaction between the discrete reinforcement and the specimen edges; effects that cannot be captured by the homogenized model.

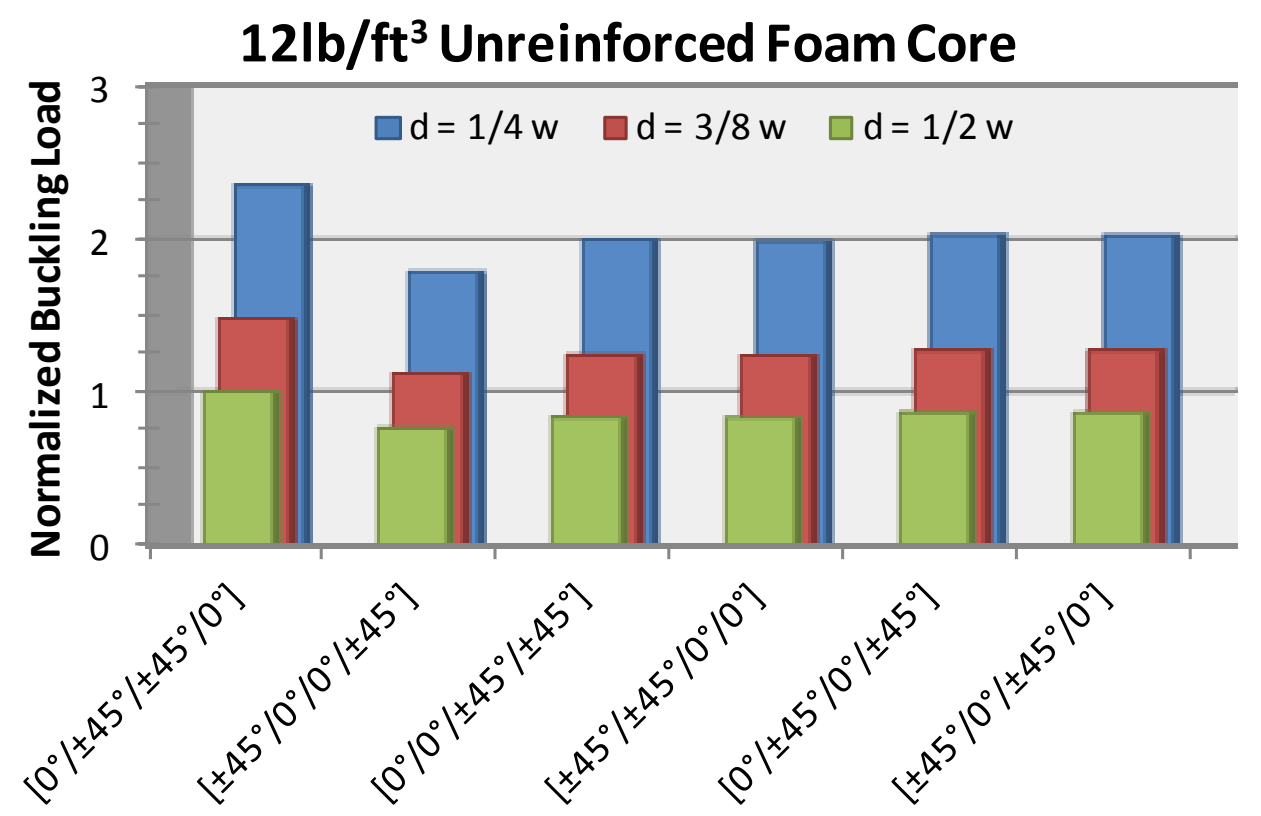

Figure 6. Effect of ply stacking sequence on normalized linear buckling loads for composite with $12 \mathrm{lb} / \mathrm{ft}^{3}$ unreinforced foam core. 


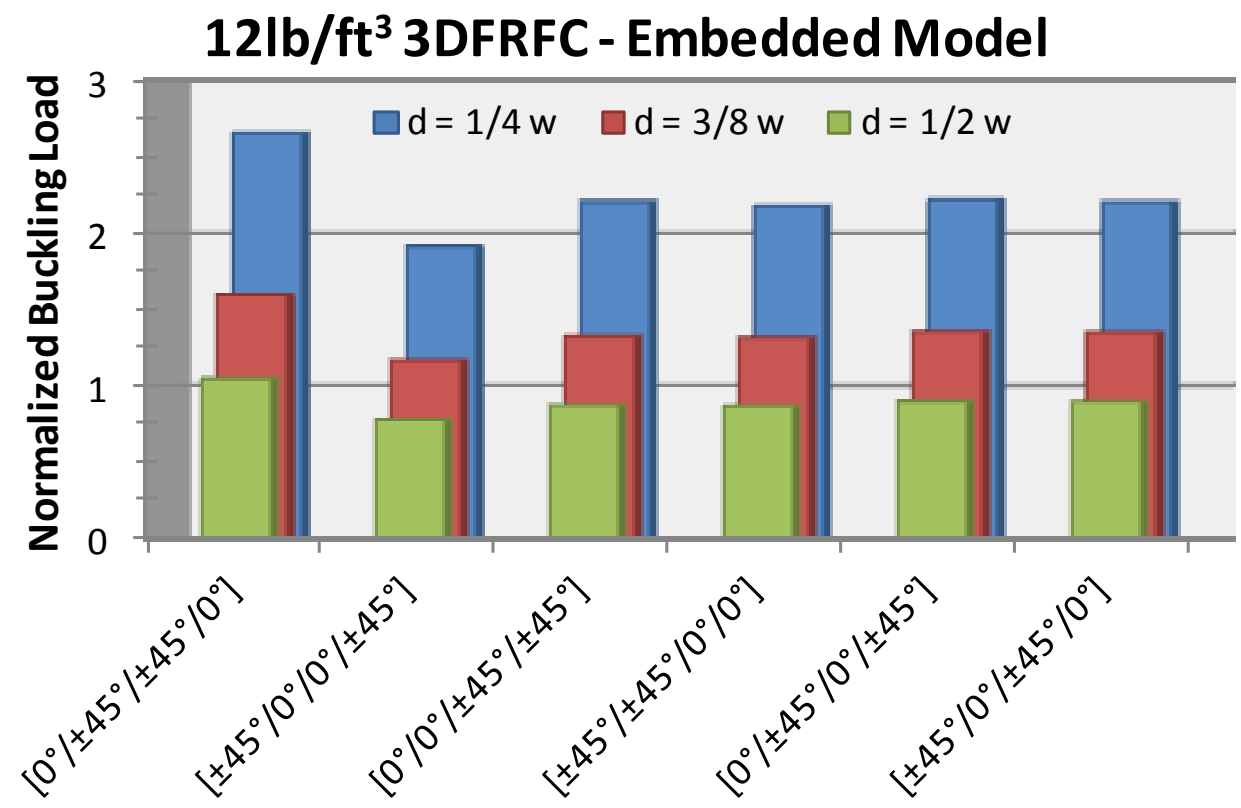

Figure 7. Effect of ply stacking sequence on normalized linear buckling loads for composite with $12 \mathrm{lb} / \mathrm{ft}^{3}$ 3DFRFC modeled with embedded elements.

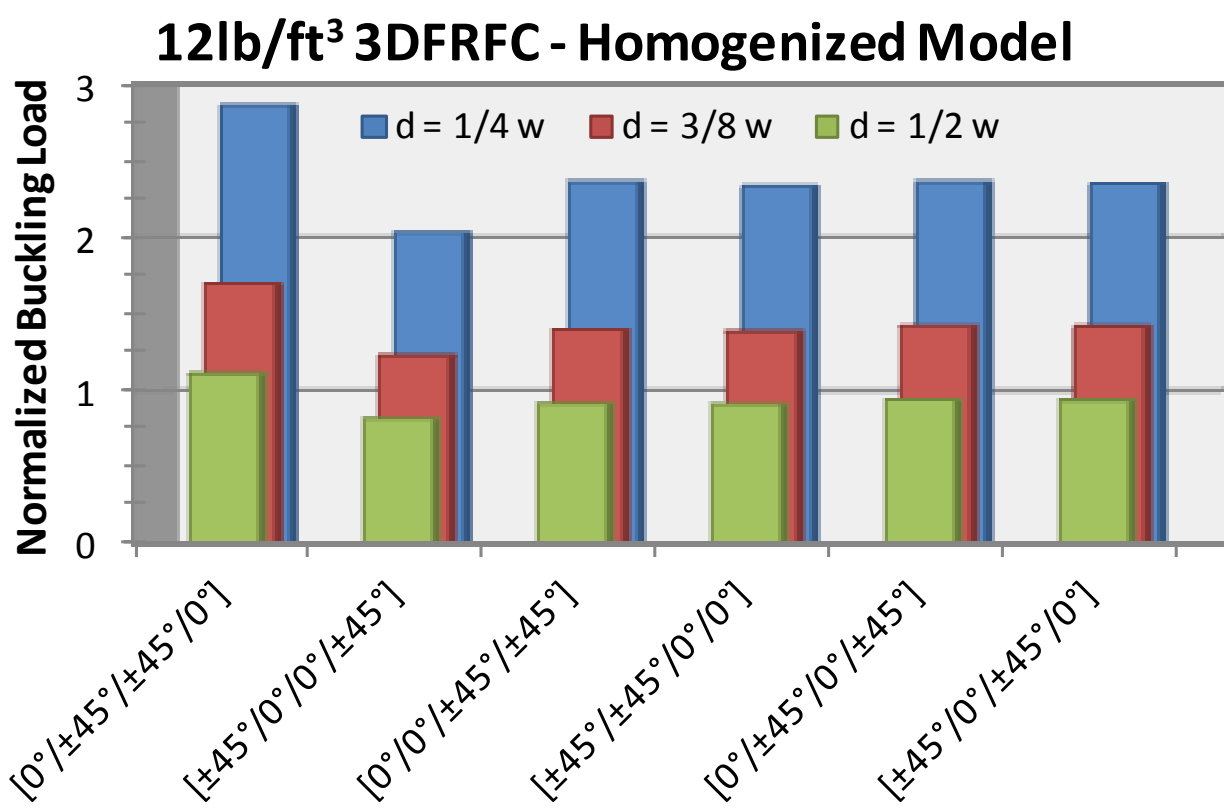

Figure 8. Effect of ply stacking sequence on normalized linear buckling loads for composite with $12 \mathrm{lb} / \mathrm{ft}^{3}$ 3DFRFC modeled with homogenized properties.

Additional insight into the local interaction of the 3DFRFC can be garnered from comparing the buckling mode displacement field of the embedded element model to that of the unreinforced foam core, Figure 9. While the overall buckling shape is similar, the 3DFRFC embedded model exhibits deformation that is highly constrained to the region of the debond. Conversely, the unreinforced foam core model exhibits a more widespread interaction with the debonded region. The higher degree of localization demonstrated by the 3DFRFC models effectively constrains the 
boundary of the debonded region and reduces the deformation of the opposing facesheet. The local constraint provided by the reinforcement near the boundary of the debond is more clearly illustrated by the deformation of the pins shown in Figure 10. This local interaction will be important to investigating the nonlinear buckling response of the EWC samples and modeling the initiation of failure.

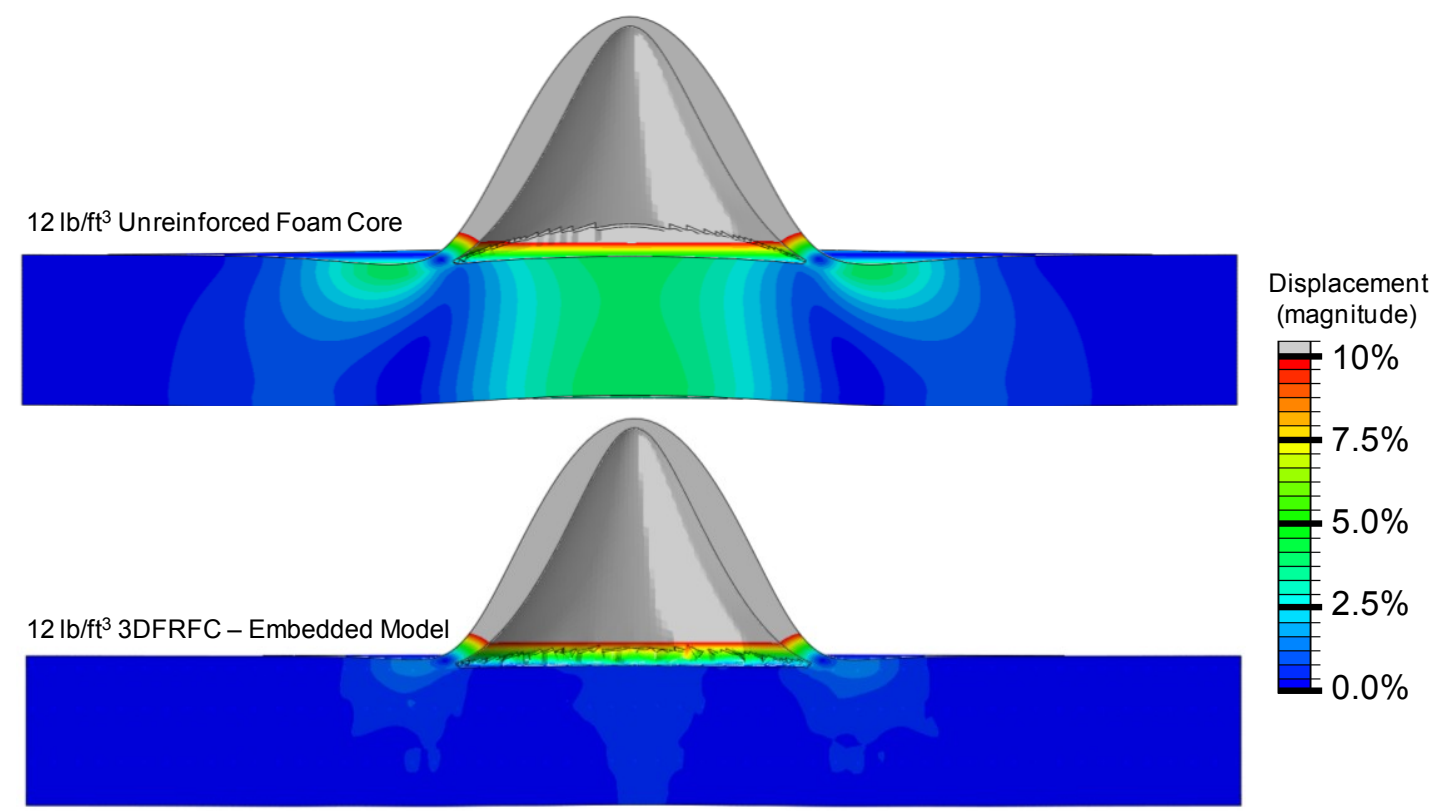

Figure 9. Displacement fields in $12 \mathrm{lb} / \mathrm{ft}^{3}$ 3DFRFC and unreinforced foam core with $\mathrm{d}=3 / 8$ $w$ and baseline facesheet ply stacking $\left[0 \% 45^{\circ} / \pm 45^{\circ} / 0^{\circ}\right]$.

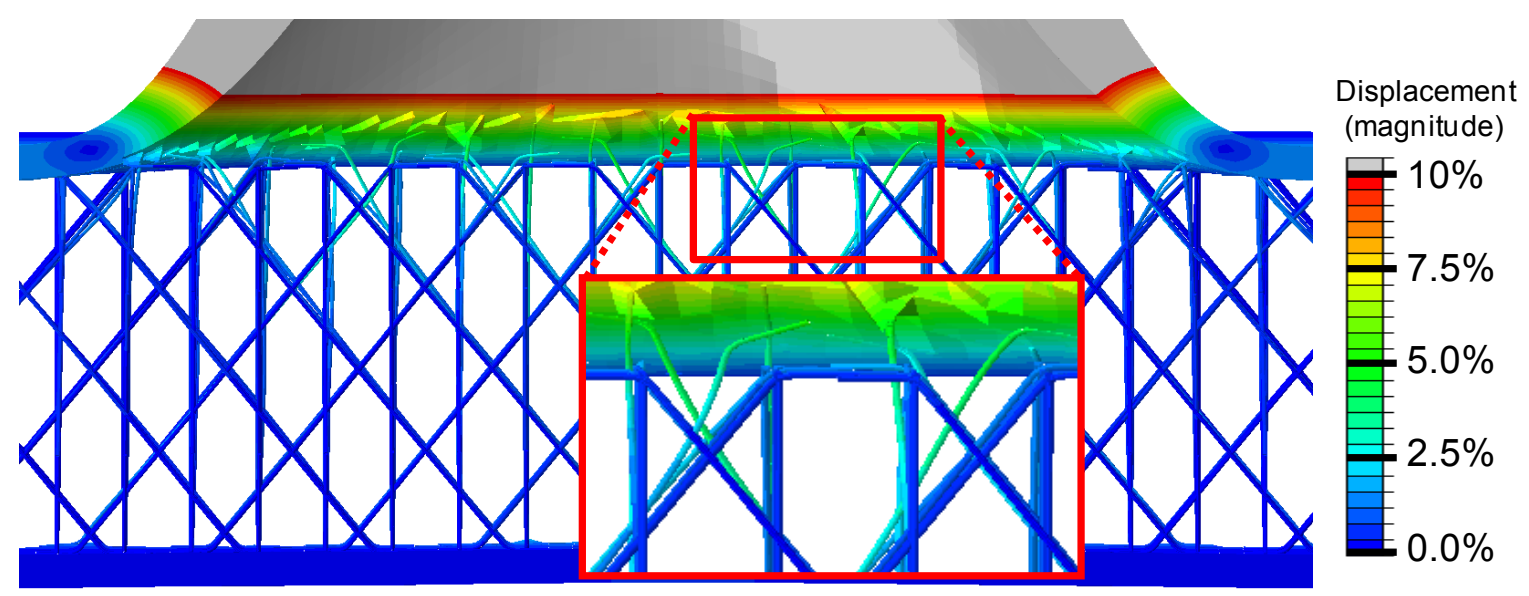

Figure 10. Local displacement fields in reinforcing pins of 3DFRFC with $d=3 / 8 \mathrm{w}$ and baseline facesheet ply stacking $\left[0 \% \pm 45^{\circ} / \pm 45^{\circ} / 0^{\circ}\right]$. 


\section{Nonlinear Response Analysis}

Linear buckling analyses were performed initially, but in some cases the linear analysis may not represent the importance of ply stacking sequence and lead to conclusions that should be verified with nonlinear response analysis. Preliminary studies were performed by running nonlinear analyses using the fundamental buckling shape from the aforementioned linear buckling analyses and applying an imperfection $1 \%$ of the total facesheet thickness. These nonlinear analyses highlight the potential for a given ply stacking sequence to have a greater influence on the global response of the debonded sandwich structure than can be understood from the linear buckling analysis alone. In the extremes of the asymmetric ply stacking sequences the local bending/extension coupling of the antisymmetric facesheet drives the delaminated region toward or away from the core within the unbonded region, Figure 11. This type of behavior could result in composite sandwiches with ply stacking sequences that show lower buckling performance in linear analysis but provide additional stability to the unbonded region. The facesheet can be effectively stabilized by forcing the unbonded region of the facesheet against the surface of the core. Additional nonlinear analysis is planned to investigate the sensitivity of this behavior to imperfection amplitude and boundary condition as well as the interaction of the discrete reinforcing microstructure of the embedded element model. Aspects related to buckling with contact, but for isotropic face sheets, are addressed in the aforementioned work by Comiez et al. ${ }^{9}$ as well as Shahwan and Waas ${ }^{10}$.

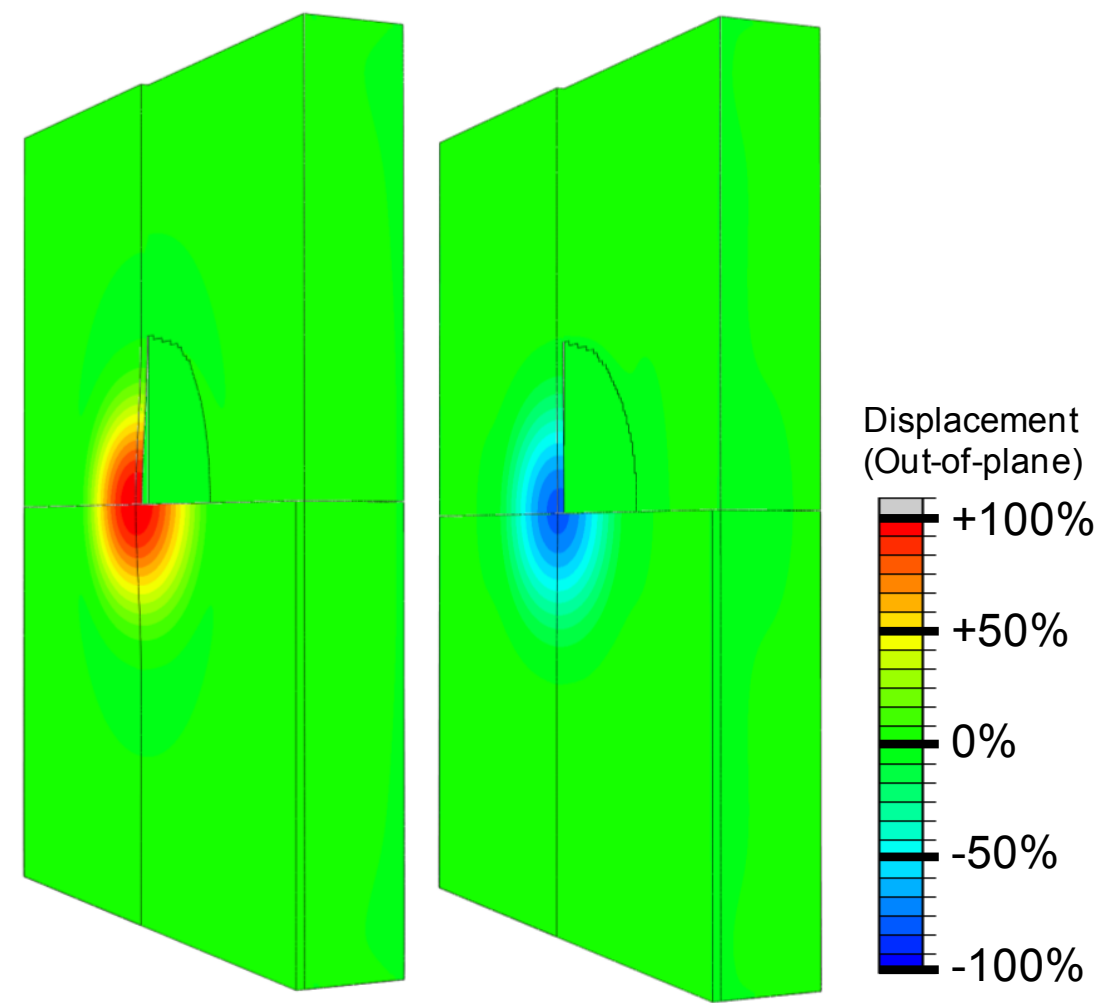

Figure 11. Through thickness displacement for $d=1 / 2 \mathrm{w}$ with homogenized 3DFRFC, $1 \%$ imperfection, and $\left[0^{\circ} / 0^{\circ} / \pm 45^{\circ} / \pm 45^{\circ}\right]$ facesheet, left, and $\left[ \pm 45^{\circ} / \pm 45^{\circ} / 0^{\circ} / 0^{\circ}\right]$ facesheet, right. 


\section{Conclusions}

Preliminary results of an investigation into the interaction of ply stacking sequence on local facesheet instability were presented. The linear buckling analysis predicted a relatively consistent effect of ply stacking between both unreinforced and reinforced core composites. The non-linear response analysis provided additional insight showing potential for antisymmetric ply stacking sequences to accelerate or suppress outward buckling of the facesheet. The ongoing nonlinear analysis will investigate the influence of this interaction on the global behavior and how the interaction varies with imperfection amplitude to hasten or delay the onset of local buckling and thus initiation of global failure. The quantification of this interaction is important to accurately model 3DFRFC sandwich structures with debonds, and to further mitigate issues related to debond growth.

\section{Acknowledgments}

The authors gratefully acknowledge The Aerospace Corporation for providing the material used for this study. This work was supported by a NASA Office of the Chief Technologist's Space Technology Research Fellowship.

\section{References}

${ }^{1}$ NidaFusion SXO/SXF, http://www.nida-core.com/spanish/fusiprod_sxosxf.htm, 2006-2008, Nida-Core Corporation.

${ }^{2}$ TYCORE® Products, http://www.webcoreonline.com/tycor\%C2\%AE-products, 2011 WebCore Technologies.

${ }^{3}$ Sandwich Structures, http://www.albint.com/businesses/aec/ProductsAndTechnologies/Pages/Sandwich-Structures.aspx, 2011 Albany International Corp.

${ }^{4}$ Z. T. Kier, A. M. Waas, J. I. Rome, V. K. Goyal, P. Schubel, G. Steckel, D. Patel, and Y. Kim, "Modeling Failure of 3D Fiber Reinforced Foam Core Sandwich Structures with Defects," 53rd AIAA/ASME/ASCE/AHS/ASC Structures, Structural Dynamics, and Materials Conference, 2012, AIAA 2012-1863.

${ }^{5}$ Z. T. Kier, J. Rome, V. Goyal, P. Schubel, G. Steckel, D. Patel, Y. Kim. Predicting Strength Reduction of Sandwich Structures with Interfacial Debonds. 25th American Society for Composites Conference, Dayton, OH (2010).

${ }^{6}$ V. Goyal, J. Rome, P. Schubel, J. Tuck-Lee, G. Steckel, and D. Patel, "Strength Evaluation of Foam Core Sandwich Structures with Butt Joints," 25th Annual Technical Conference of the American Society for Composites. September 20-23, 2010, Dayton, OH.

73M Scotch-Weld Structural Adhesive Film AF 191, March 2009, Technical Datasheet, St. Paul, MN.

${ }^{8}$ T. Liu., Z. Deng, and T. Lu, “Analytical Modeling and Finite Element Simulation of the Plastic Collapse of Sandwich Beams with Pin-reinforced Foam Cores,” International Journal of Solids and Structures, 45, 2008, pp. 5127-5151.

${ }^{9}$ J. Comiez, A. Waas and K. Shahwan, "Delamination Buckling: Experiment and Analysis" Int. J. Solids and Structures, vol. 32, No. 6/7, pp767-782, 1995.

${ }^{10}$ K. Shahwan and A. Waas, "A Mechanical Model for the Unilateral Buckling of Rectangular Plates," Int. J. of Solids \& Structures, Vol. 31, No.1, pp75-89, 1994.

*All trademarks, service marks, and trade names are the property of their respective owners 